4-3-2018

\title{
The Extent of Tebuconazole Leaching from Unpainted and Painted Softwood
}

Klara Kukowski

Joshua Hatton

Evguenii I. Kozliak

Alena Kubotava

University of North Dakota, alena.kubatova@und.edu

How does access to this work benefit you? Let us know!

Follow this and additional works at: https://commons.und.edu/chem-fac

\section{Recommended Citation}

Klara Kukowski, Joshua Hatton, Evguenii I. Kozliak, et al.. "The Extent of Tebuconazole Leaching from Unpainted and Painted Softwood" (2018). Chemistry Faculty Publications. 8.

https://commons.und.edu/chem-fac/8

This Article is brought to you for free and open access by the Department of Chemistry at UND Scholarly Commons. It has been accepted for inclusion in Chemistry Faculty Publications by an authorized administrator of UND Scholarly Commons. For more information, please contact und.commons@library.und.edu. 


\section{The Extent of Tebuconazole Leaching from Unpainted and Painted Softwood}

Klara Kukowskia , Joshua Hatton ${ }^{a}$, Evguenii I. Kozliakª Alena Kubátováa

a Chemistry Department, University of North Dakota, Grand Forks, ND, 58202, USA

Submitted to December 2017 to Science of the total Environment 633 (2018) 1379-1385

https://doi.org/10.1016/i.scitotenv.2018.03.274

\section{Highlights}

- High humidity environment did not impact sorbed tebuconazole (TAZ) in wood

- Simulated continuous rain led to moderate TAZ leaching from the end grain

- End-grain sealing with water-based paint reduced TAZ depletion two-fold

- Simulated intermittent rain led to additional TAZ loss not caused by leaching

\section{Abstract}

Exposure to water and high air humidity may affect the preservation of wood products as many preservatives are water-soluble and thus likely to leach. In this study, depletion of a common fungicide, tebuconazole (TAZ), from treated wood was investigated using a ${ }^{14} \mathrm{C}$-labeled tracer. The wood species and treatment technique were chosen to be representative of products such as windows and doors; specifically, ponderosa pine was dip treated with a solvent-based, metal-free formulation. The impact of different aqueous settings including high air humidity, and either simulated continuous or intermittent rain was evaluated over a period of two months. Along with the exposure type, the effect of end-grain sealing on TAZ loss was explored.

Despite the exposure of treated wood to laboratory-simulated harsh environmental conditions, more than $60 \%$ of the originally sorbed TAZ remained in the wood under all 
scenarios. While high air humidity did not lead to TAZ depletion, simulated continuous rain led to a TAZ leaching mainly from the end grain. TAZ leaching was found to be higher for unpainted wood, where up to $40 \%$ of the originally sorbed TAZ was prone to depletion from an end grain. End-grain sealing with water-based primer and paint led to a substantial two-fold reduction of TAZ leaching. Unexpectedly, wood exposure to intermittent rain caused additional TAZ loss that could not be explained only by water leaching.

\section{Keywords}

tebuconazole; leaching; wood; rain; paint; fungicide

\section{Introduction}

Wood is a renewable material widely used in residential and commercial construction due to its appearance, strength and good insulating properties among other qualities. To increase its lifespan and durability, wood is commonly treated with inorganic and organic preservatives, often in combination, to achieve protection against a broad range of microorganisms. When used as a building material, wood is regularly exposed to water in the form of high air humidity and rain, which can mobilize sorbed preservatives and lead to their leaching. Understanding the kinetic of this process is essential to prevent wood deterioration. ${ }^{1}$ While the leaching behavior of inorganic preservatives has been extensively studied, ${ }^{2-4}$ leaching of organic preservatives has been investigated to a lesser extent, and was limited to pressure-treated wood using mainly metal-containing formulations.$^{5-10}$ Triazoles such as tebuconazole (TAZ) and 
propiconazole are among the most frequently used organic wood preservatives, ${ }^{11-15}$ thus determination of their leaching potential under various conditions is warranted.

The leaching potential of preservatives was shown to depend on various parameters, such as affinity to wood in terms of sorption and fixation. ${ }^{16-18}$ In general, the leaching process is characterized by preservative partitioning between the wood and the aqueous phase (mobilization), followed by its diffusion through the wood porous matrix. ${ }^{5}$ Studies performed by Lupsea et al. using a copper-borate-azole formulation showed that TAZ fixation within wood was $\mathrm{pH}$ dependent and thus suggested hydrogen bonding of TAZ with the carboxyl and phenolic groups of wood. ${ }^{6-7}$ TAZ leaching likely occurred through its molecular association and co-elution with wood extractives, such as carboxylic acids, alcohols, phenols, and ketones. ${ }^{5,7}$ TAZ was also found to be physically dislodged from pressure-treated wood by rain as opposed to its dissolution in water. ${ }^{7,} 19$

Previous studies focusing on TAZ leaching mainly used wood co-treated with copper and other metals; ${ }^{5-10}$ however many wooden products, such as windows, are treated with formulations that do not contain metals. Since complexation with a metal has been shown to reduce TAZ leaching rates, ${ }^{20-22}$ the published results cannot be used to describe metal-free systems. For instance, the natural depletion of TAZ from pine decks co-treated with copper during a ten-month exposure to rain was found to be between $0.8-1.4 \%$ of the original amount present in decks. ${ }^{23}$ In contrast, a year-long leaching study on treated spruce using a formulation without metals showed the percentages of leached TAZ to be $21.0-25.5 \%$ in the first 7 months and $22.5-30.3 \%$ in 12 months. ${ }^{19}$ 
In addition to using metals as part of the formulation, the majority of studies were performed on pressure-treated wood that was fully immersed into water. ${ }^{6,}$ 9-10, 16 Pressure treatment of wood (e.g., wood impregnation with preservatives under pressure) is commonly used for ground contact wood (e.g., decks) to increase the penetration depth and active loading. ${ }^{23}$ However, in North America, nearly all of the wood used to produce windows and door frames is dip treated, ${ }^{13}$ which is cost-effective and ensures sufficient concentration of preservatives near the wood surface. Moreover, full immersion of treated wood into water is not a real-world scenario descriptive of window exposure to rain, where only the surface is exposed to water droplets. Schoknecht et al. have confirmed this assertion by comparing the propiconazole leaching from treated materials via full immersion into water to water spraying onto the surface (simulated rain) and found that the initial preservative emissions were much higher for the immersion experiments than those obtained with simulated rain. ${ }^{16}$

In our previous work we've demonstrated a TAZ depletion from painted window frame sections to occur primarily from the end grain, which amounted to $\sim 20 \%$ of the originally sorbed TAZ when exposed for six months to harsh environmental conditions. ${ }^{24}$ Leaching was found to be the major route of TAZ losses, even though vaporization was shown to affect the sorbed triazoles as well. Whether the observed TAZ losses after six months were final or would increase with longer exposures was uncertain. The impact of paint application was not addressed either. Therefore, this study aims to complement the existing data on TAZ leaching from treated wood by exposing sealed and unsealed wood to laboratory-simulated outdoor conditions. 
Triazole leaching from painted and unpainted softwood was monitored in laboratory settings for two months using a tracer, ${ }^{14} \mathrm{C}$-labeled TAZ. The wood species and treatment technique were designed to be representative of windows and doors; specifically, ponderosa pine was dip treated with a solvent-based metal-free formulation. Treated wood was exposed to various conditions, such as high air humidity, simulated continuous and intermittent rain. The effect of exposure and paint application was evaluated by analyzing both the exposed wood and run-off water.

\section{Materials and Methods}

\subsection{Chemicals}

Uniformly ${ }^{14} \mathrm{C}$-labeled TAZ obtained from the Commerce Institute of Isotopes Co., Ltd. (Budapest, Hungary) with a radioactivity of $7.4 \mathrm{MBq} \mathrm{mL}^{-1}$ was spiked as a tracer to a commercial wood formulation, Woodlife 111 RTU from Kop-Coat, Inc. (Pittsburgh, PA, USA), resulting in a radioactivity of $0.74 \mathrm{MBq} \mathrm{L}^{-1}$ and TAZ concentration of $0.12 \mathrm{~g} \mathrm{~L}^{-1}$. A scintillation cocktail, Betamax, was obtained from MP Biomedicals, LLC (Solon, OH, USA) and acetone (GC grade) were purchased from Fisher Scientific (Pittsburgh, PA, USA). The applied exterior wood primer was from Akzo Nobel Coatings Inc. (High Point, NC, USA), and white paint was made by The Valspar Corporation (Minneapolis, MN, USA). Both the primer and paint were water-based.

\subsection{Wood Treatment}

Size-reduced samples $\left(5.0 \times 2.5 \times 1.5 \mathrm{~cm}^{3}\right)$ of conditioned ponderosa pine wood were dip treated with the radiolabeled Woodlife formulation for 1.0 min resulting in a 
${ }^{14} \mathrm{C}$-TAZ sorption of $\sim 55 \mathrm{ng}$ per wood sample. The repeatability of such wood treatment was previously shown to be high, especially for dipping times exceeding $0.5 \min ^{25}$ Wood was then placed on wooden skewers and dried in a hood for 24 hours. Half of the samples were directly placed into the chambers while the other half were coated on all sides using a foam brush with one layer of the primer and two layers of paint, where each coating was followed by drying for 24 hours before the next layer was applied.

\subsection{Wood Exposure}

Experiments were performed in triplicate using painted and unpainted wood. The exposure conditions included low humidity, high humidity, continuous leaching (i.e., continuous rain), and weekly alternating leaching and drying (i.e., intermittent rain). Experimental details are provided in Table 1. Control samples consisted of treated wood blocks wrapped in aluminum foil that were placed in an enclosed bag into a freezer (e.g, to prevent any TAZ evaporation and leaching).

Table 1. Experimental layout including the exposure types with corresponding chamber conditions. For all experiments, conditioned ponderosa pine wood was dip treated with Woodlife containing ${ }^{14} \mathrm{C}-\mathrm{TAZ}$.

\begin{tabular}{ccccc}
\hline Wood Type & Exposure Type & $\begin{array}{c}\text { Temperature } \\
{\left[{ }^{\circ} \mathrm{C}\right]}\end{array}$ & $\begin{array}{c}\text { Relative } \\
\text { Humidity [\%] }\end{array}$ & $\begin{array}{c}\text { Exposure } \\
\text { Time [weeks] }\end{array}$ \\
\hline Unpainted & Freezer control & -18 & - & 8 \\
\hline Unpainted & Low humidity & $\mathrm{rt}^{*}$ & 15 & 8 \\
\hline Unpainted & High humidity & $\mathrm{rt}$ & 80 & 8 \\
\hline $\begin{array}{c}\text { Unpainted/ } \\
\text { Painted }\end{array}$ & $\begin{array}{c}\text { Continuous leaching } \\
\text { (continuous rain) }\end{array}$ & $\mathrm{rt}$ & 15 & 8 \\
\hline
\end{tabular}




\begin{tabular}{ccccc}
\hline $\begin{array}{c}\text { Unpainted/ } \\
\text { Painted }\end{array}$ & $\begin{array}{c}\text { Leaching/drying } \\
\text { (intermittent rain) }\end{array}$ & $\mathrm{rt}$ & 15 & 10 \\
${ }^{*} \mathrm{rt}=$ room temperature & & &
\end{tabular}

For low and high humidity conditions, individual wood samples were placed in separate glass chambers and flushed weekly with air while maintaining the desired humidity. For leaching experiments, wood was placed on a platform under a $50^{\circ}$ angle with part A being at the top. Deionized water was dripped onto the transversal surface of part $A$ (at a flow of ca. $0.1 \mathrm{~mL} \mathrm{~min}^{-1}$ ) and descended towards part $\mathrm{D}$ and then into the run-off collection flask, as illustrated in Figure 1. As a result of this design, several water droplets were continuously accumulating near the end-grain side of part D. For the intermittent rain, wood blocks were periodically transferred into an enclosed chamber after a week of leaching and left to dry for another week.

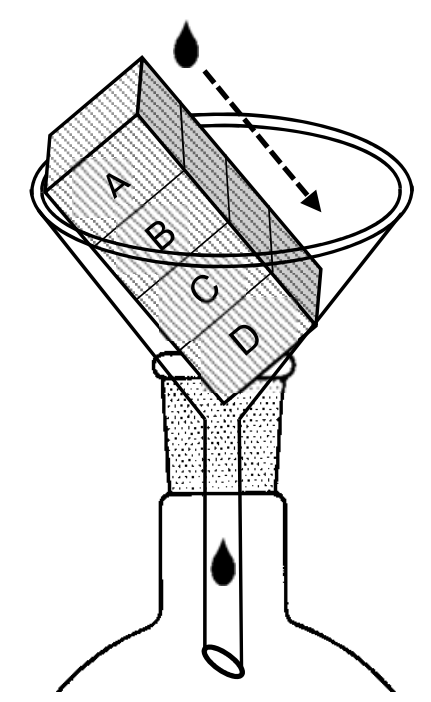

Figure 1. Experimental design of TAZ-treated wood exposure to simulated rain. 


\subsection{Extraction \& Analysis}

After the exposure, wood samples were sheared with a hammer and knife into four equal parts $\left(\sim 1.25 \times 2.5 \times 1.5 \mathrm{~cm}^{3}\right)$, where parts $A$ and $D$ were those originally located next to the end-grain side whereas parts $B$ and $C$ were the two middle sections (Figure 1). Each wood part was further sheared into smaller pieces to allow for an exhaustive TAZ extraction, which was carried out for 16 hours using a Soxhlet apparatus with 130.0 $\mathrm{mL}$ of acetone. The extract was evaporated to dryness using a vacuum rotary evaporator and reconstituted in $1.5 \mathrm{~mL}$ of acetone. For the liquid scintillation counting analysis, $1.5 \mathrm{~mL}$ of an acetone dissolved sample was vortexed with $3.5 \mathrm{~mL}$ of the scintillation cocktail and analyzed after an overnight equilibration in a scintillation counter, Beckman Coulter LS 6500, purchased from Beckman Coulter, Inc. (Fullerton, CA, USA). The analysis was run in duplicate for $10.0 \mathrm{~min}$ in a DPM calculation mode (disintegrations per minute, which is directly proportional to the tracer concentration).

Water run-off was collected several times per week and the volume was recorded. As with the wood extracts, run-off water was evaporated to dryness using a vacuum rotary evaporator and reconstituted in $1.5 \mathrm{~mL}$ of acetone, which was analyzed using a scintillation counting analysis as described previously.

\subsection{Maximum Leachable TAZ Calculation}

TAZ leaching curves were plotted as the amount of applied water onto wood versus the cumulative leached TAZ amount. Based on the perceived analogy to Langmuir isotherm, the leaching can be described by Eq. 1, where $\left[T A Z_{W}\right]$ referred to the cumulative amount of leached TAZ, $\left[\mathrm{H}_{2} \mathrm{O}\right]$ was the amount of applied water, $\left[\mathrm{TAZ}_{\mathrm{MAX}}\right]$ was the highest TAZ amount that could be leached from treated wood, and $K$ 
represented an affinity constant. In the given form of Eq. $1, K$ reflects the TAZ affinity to wood whereas the affinity to water would be reflected by the inverse value of this constant.

Eq. 1

$$
\left[T A Z_{W}\right]=\left[T A Z_{M A X}\right] \times \frac{\left[\mathrm{H}_{2} \mathrm{O}\right]}{K+\left[\mathrm{H}_{2} \mathrm{O}\right]}
$$

In order to calculate $\left[T A Z_{M A X}\right]$, sigmoidal curves were transformed into a linear function by plotting $\left[\mathrm{H}_{2} \mathrm{O}\right]^{-1}$ on the $x$ axis and $\left[\mathrm{TAZ}_{W}\right]^{-1}$ on the $y$ axis (Eq. 2), where the intersection of this linear function with the $y$ axis represented $\left[T A Z_{M A X}\right]^{-1}$. The slope of the function was defined by $K /\left[T A Z_{M A X}\right]$.

Eq. 2

$$
\frac{1}{\left[T A Z_{W}\right]}=\frac{K}{\left[T A Z_{M A X}\right]} \times \frac{1}{\left[H_{2} O\right]}+\frac{1}{\left[T A Z_{M A X}\right]}
$$

\section{Results and Discussion}

In order to monitor the leaching extent of triazole fungicides from painted and unpainted softwood, ${ }^{14} \mathrm{C}$-labeled TAZ was used as a fungicide tracer. The overall impact of the several aqueous environmental exposures shows no losses under either of two extreme humidity settings, 15 and $80 \%$ (Figure 2). By contrast, when wood was exposed to the simulated continuous rain conditions, a moderate TAZ depletion was found to occur from the end grain (14-33\%) thus being attributed to leaching. As expected, lower TAZ leaching was observed in painted wood. The mass balance was not closed for intermittent rain conditions suggesting that the additional TAZ loses may have occurred due to water enhanced vaporization. Nevertheless, the experimental setup allowed for the investigation of mechanistic aspects of TAZ leaching, providing the insights on spatial losses from wood and the leaching kinetics. 


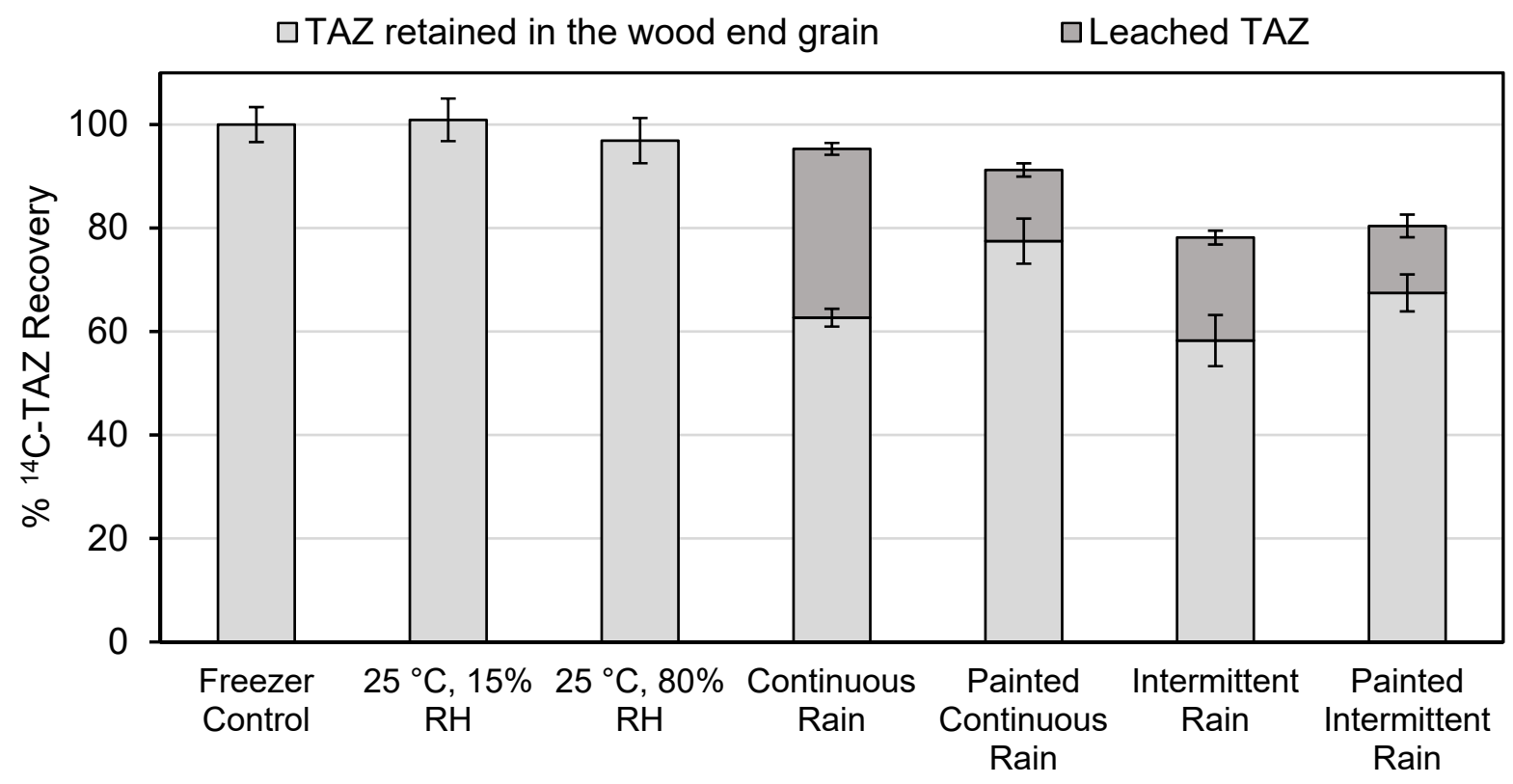

Figure 2. Percent ${ }^{14} \mathrm{C}-\mathrm{TAZ}$ recoveries from end grains of painted and unpainted treated wood exposed to various humidity and simulated rain conditions, complemented by ${ }^{14} \mathrm{C}$ TAZ recoveries from the run-off water. $\mathrm{RH}$ denotes relative humidity.

\subsection{TAZ Distribution and Recoveries from Wood End Grain and Middle Sections}

The TAZ amounts recovered from the parts located next to the end grain (i.e., Figure 3, parts $A$ and $D$ ) were significantly higher than those found in the middle sections (i.e., parts $B$ and $C$ ) due to a significant contribution of longitudinal TAZ penetration. By contrast, the middle sections contained TAZ sorbed only via the transversal diffusion. This is consistent with the previously shown TAZ distribution in wood as a result of dip treatment, where the sorption in the transversal direction distributed smaller amounts of TAZ evenly onto the wood surface within a $0.5 \mathrm{~cm}$ depth, while the diffusion in the 
longitudinal direction mediated its large deposition in the first $0.2 \mathrm{~cm}$ from the end grain. ${ }^{25}$

As shown above the exposure to low and high humidity did not seem to impact the sorbed TAZ, while exposure to simulated rain led to a sizable TAZ depletion from end grain surfaces. The less pronounced TAZ loss from part $A$ (compared to $D$ ) was due to the water application, where only the transversal surface was exposed to the simulated rain (the end-grain side remained untouched), which was not sufficient to cause TAZ leaching. Conversely, the end grain of part D was fully accessible to water and became soaked into the aqueous phase after a few days of exposure, which led to TAZ leaching. For both unpainted and painted wood exposed to intermittent rain, a moderate TAZ depletion was observed from part A as well, and these losses are discussed further in the next section with respect to TAZ leaching kinetics.

Even though the TAZ recoveries from the middle sections (i.e., parts $B$ and $C$ ) varied between exposures (Figure 3), the differences were likely caused by the wood structure and the accumulation of minor losses during the wood treatment rather than by the exposures. Small variations in treatment time and the wood structure affect the depth of fungicide penetration as opposed to the fungicide deposition at the end grain.

Therefore, the recovered TAZ amounts from parts $B$ were compared to parts $C$ of wood exposed to identical conditions rather than to wood exposed to different conditions. Since no statistical difference was found among these two parts for each exposure, the varying TAZ recoveries were not deemed to be results of humidity and water exposures. A similar trend was observed previously, where wood environmental exposure led to the TAZ depletion exclusively from the end grain. ${ }^{24}$ 


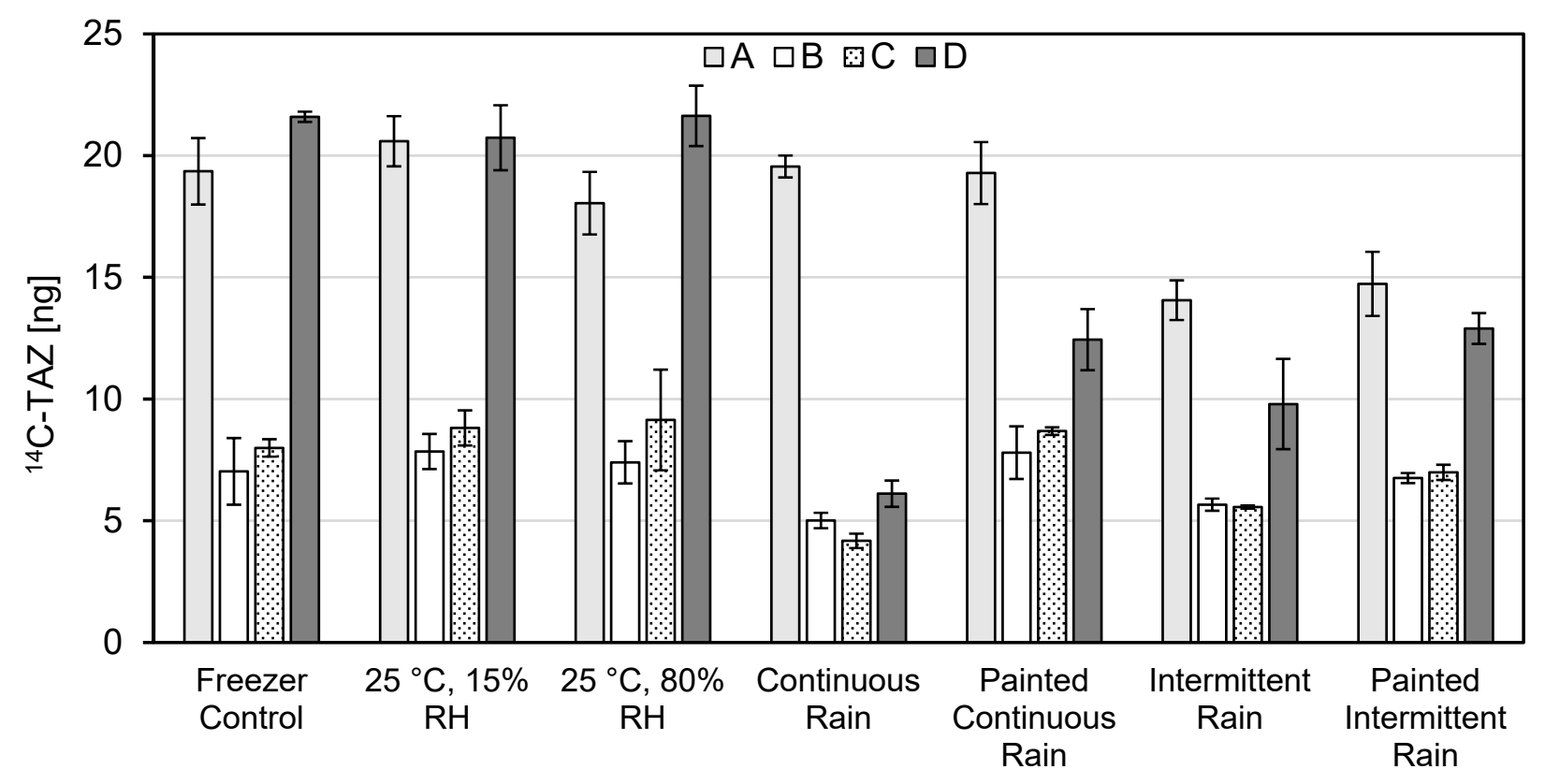

Figure 3. Distribution of ${ }^{14} \mathrm{C}-\mathrm{TAZ}$ recovered from the exposed wood.

\subsection{TAZ Leaching Kinetics}

The TAZ leaching rate was investigated with respect to the amount of water applied on the wood surface and was found to be the highest for the unpainted wood exposed to continuous rain (Figure 4). After two months of continuous rain exposure, $13.5 \mathrm{ng}$ of TAZ leached from the unpainted wood representing $\sim 25 \%$ of the originally sorbed TAZ. The paint application reduced the leached TAZ amount to $6.0 \mathrm{ng}(\sim 11 \%)$. The observed reduction of TAZ leaching from painted wood can be explained by the effect of coatings, such as paints and primers, which is expected to reduce the water transport in and out of wood by creating a physical barrier that partially repels water. The rate of water sorption was previously shown to be the highest for uncoated wood, followed by wood painted with a water-based coating and slowest for wood painted with a solvent-based 
coating. ${ }^{26}$ Likewise, end-grain sealing is commonly employed in industry to reduce the decay of windows, which is often initiated in the end grain within joints. ${ }^{27}$

$\Delta$ Painted Continuous Rain $\Delta$ Painted Intermittent Rain $\|$ Intermittent Rain $\square$ Continuous Rain

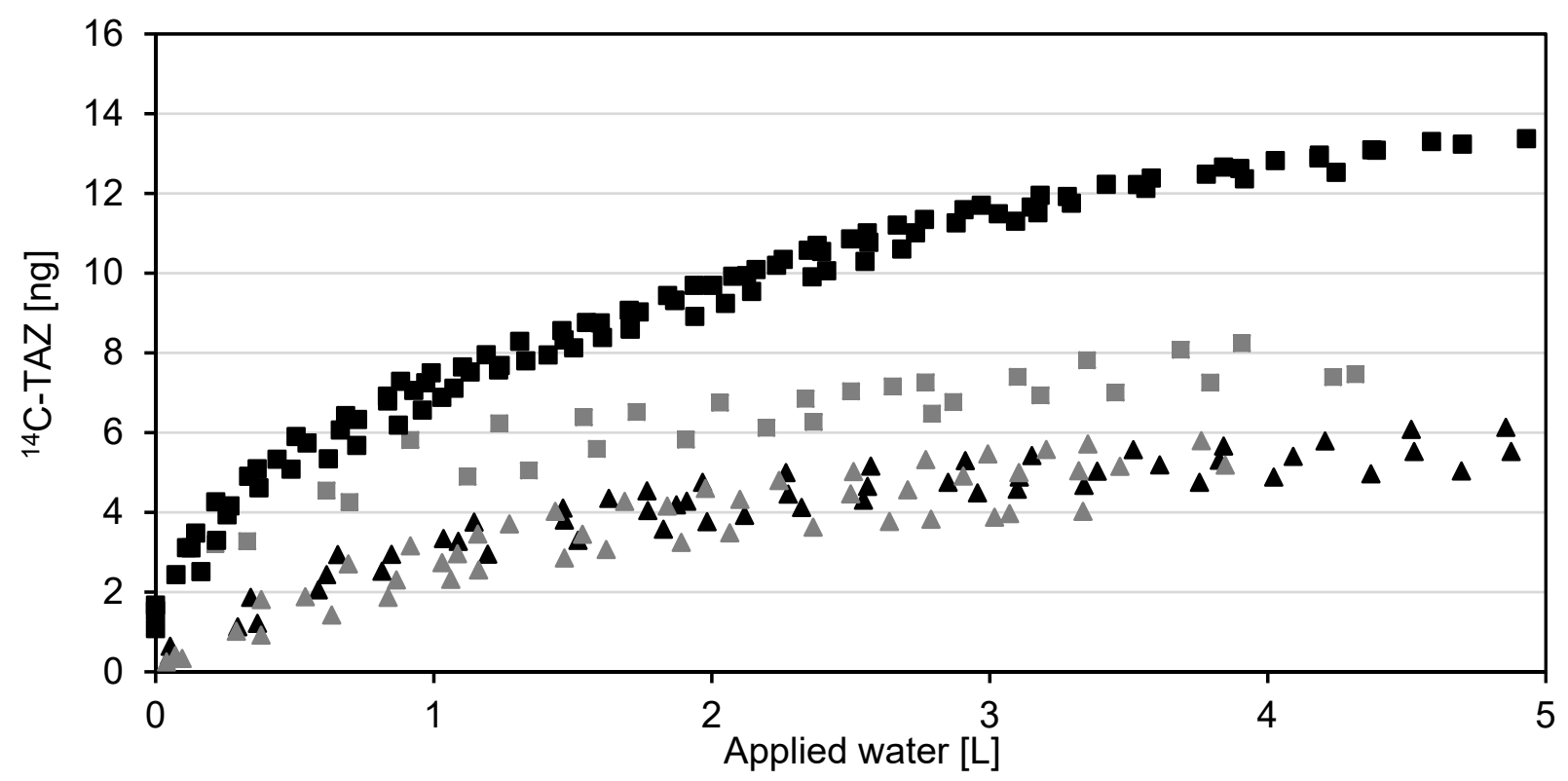

Figure $4 .{ }^{14} \mathrm{C}-\mathrm{TAZ}$ recovered in the collected run-off water from unpainted and painted wood exposed to simulated continuous and intermittent rain.

Wood exposure to intermittent rain led to a reduced TAZ leaching rate from unpainted wood and similar leaching rate from painted wood compared to the continuous rain (Figure 4). Since wood saturation with water is essential for fungicide leaching ${ }^{28}$ wood had to be re-saturated after each drying period for leaching to occur. However, with unlimited time, the intermittent rain exposure of unpainted wood would result in the same leached amount of TAZ as the continuous rain. In the case of painted wood, drying intervals did not impact the TAZ leaching kinetics since water transport in 
and out of wood was reduced, which resulted in ineffective wood drying between the leaching periods hence fast re-saturation.

In addition, intermittent rain exposure led to the TAZ depletion from wood parts A, which was not the case for continuous rain. Furthermore, this additional TAZ loss was not reflected in the run-off water analysis and was thus not caused by leaching. For painted wood, the TAZ leaching curves observed for continuous and intermittent rain were similar (Figure 4) as well as the recovered TAZ amounts from wood parts $D$ (Figure 3), suggesting that TAZ presumably leached from part $\mathrm{D}$. The same trend was observed for unpainted wood, where the leaching rate was higher for continuous rain exposure and consequently the TAZ amount recovered from wood part D was lower compared to the intermittent rain exposure.

This unexpected TAZ loss from wood part A was seen only after introducing drying intervals between water exposures, therefore we hypothesize that TAZ co-evaporated from wood with water. When wood was saturated with water, TAZ was depleted exclusively through leaching as water completely sealed the pores and hindered evaporation. However, as wood started drying, the pores were no longer fully saturated so the association with water (possibly via hydrogen bonding) enabled TAZ to evaporate along with water (enhanced vaporization). Since the TAZ depletion from wood part A was not observed when wood was exposed to $80 \% \mathrm{RH}$, we assume that the TAZ association with water occurred only when pores were first saturated. Considering the previously reported TAZ evaporation rate from the wood end grain at $25^{\circ} \mathrm{C}, \sim 1 \mu \mathrm{g} \mathrm{m}^{-2} \mathrm{~h}^{-1},{ }^{24}$ the TAZ amount that could have co-evaporated with water during 
the five weeks was $\sim 4 \mathrm{ng}$. This amount would correspond to $\sim 7 \%$ of the initially sorbed TAZ and allow for the mass balance closure for intermittent rain conditions.

\subsection{TAZ Depletion from Wood Exposed to the Outdoor Environment}

In our previous study, the TAZ depletion from painted softwood after a six-month environmental exposure (involving high humidity, rain, wind, etc.) was found to be $\sim 20 \%$ of the originally sorbed TAZ; however, it was unclear whether these losses were final or would increase with the exposure time ${ }^{24}$ The present study shows that even though a portion of TAZ is prone to be leached from the end grain possibly due to its weak fixation within the wood matrix, this amount is limited as evident from the leaching curve levelling off for each exposure type. In order to estimate the highest TAZ amount that can be leached from treated wood, results from continuous leaching exposure were transformed into a linear relationship (Figure 5) as described in Section 2.5. For unpainted wood, the maximum leachable TAZ amounted to $22 \mathrm{ng}$ or $40 \%$ of the originally sorbed amount, while for painted wood, the maximum leachable TAZ was calculated to not exceed $12 \%$ (6.7 ng). These results corresponded to TAZ leached from one wood end-grain side (wood part $D$ ), therefore, the maximum leachable TAZ would double for exposures where leaching from both sides was plausible. Besides the change in $T A Z_{M A X}$, the affinity constant $K$ also decreased for painted wood ( $K$ was 3.7 for unpainted compared to 0.5 for painted wood) suggesting that $K$ represents the affinity of TAZ for wood, where low values indicate higher affinity. 


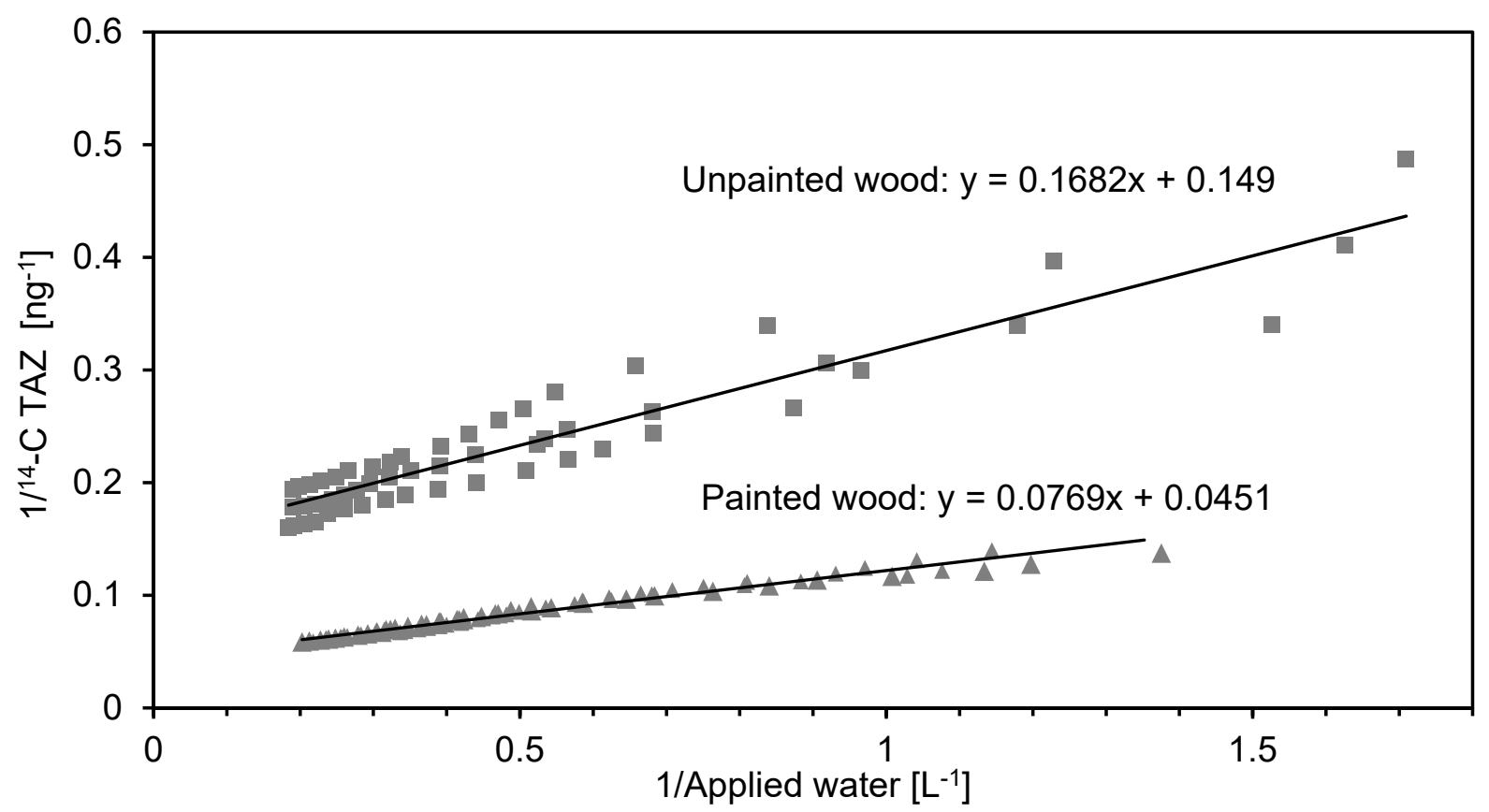

Figure 5. Determination of the highest TAZ amount that can be leached from one wood end grain.

During the outdoor study, ${ }^{24}$ painted wood was exposed to rain and dry weather conditions and the leaching was facilitated only via one wood side, which are similar settings to the wet/dry cycles exposure used in this study. The observed $(20 \pm 10) \%$ TAZ loss in the wood exposed to the outdoor environment is also similar to the TAZ loss found during the current indoor study, thus demonstrating the validity of laboratory measurements.

\subsection{Impact of TAZ Loss on Wood Protection}

TAZ that is depleted from wood over time does not contribute significantly to environmental pollution, but is important from the product perspective as it may affect the wood integrity if the preservative loss is extensive. The TAZ amounts added to wood 
formulations $(0.21 \%)^{29}$ are one to two orders of magnitudes smaller than those present in agricultural mixtures that are intended for crop protection $(7-50 \%)^{30-31}$. Additionally, a window frame absorbs a similar volume of formulation as is typically sprayed on a land size of only 4 acres. Therefore, the TAZ amount that is depleted from wood over its lifetime does not significantly contribute to environmental pollution, but may affect the durability and quality of wood products. In this study, more than $75 \%$ of TAZ was shown to remain in the painted wood despite the exposure to water. TAZ was depleted mainly from the end grain, which contained the highest amount of sorbed TAZ. Despite the loss, a significant TAZ amount stayed in this region - either greater or similar TAZ amount as was in the inner wood parts, thus the wood remained protected. On the other hand, since the end grain represents the most vulnerable part of windows and the maximum leachable TAZ amount for an unpainted end grain was shown to be $40 \%$ of the initially sorbed amount, any means of end grain protection, such as sealing with a primer and paint, would be beneficial and enhance wood protection across its lifespan.

\section{Conclusions}

Despite an eight-week exposure of treated wood to laboratory-simulated environmental conditions, more than $75 \%$ of originally sorbed ${ }^{14} \mathrm{C}-\mathrm{TAZ}$ remained in the wood for all exposure types. While the high humidity environment did not impact the sorbed TAZ, water application led to a TAZ depletion mainly from the end grain. Since this region initially contained the highest TAZ amount, the loss did not impact the overall wood protection as a sufficient amount persisted in all wood parts. 
The TAZ leaching rate was found to be the highest for unpainted wood exposed to simulated continuous rain. Upon extensive water application, up to $40 \%$ of the originally sorbed TAZ may leach from end grain based on the obtained kinetic data. The TAZ leaching rate from unpainted wood exposed to intermittent rain declined since wood was allowed to dry between weeklong rain intervals, yet it might eventually result in a comparable TAZ loss. Additionally, primer and paint coatings led to a substantial reduction of TAZ depletion with the maximum leachable TAZ fraction of $12 \%$. Therefore, end-grain sealing was found to be beneficial as it enhanced the wood protection in the long run by acting as a physical barrier and decreasing the wood affinity for water.

\section{Acknowledgements}

Marvin Windows and Doors is acknowledged for financial support. Authors would like to thank Ben Wallace and Stephen Fisher for stimulating discussions. 


\section{References}

1. Goodell, B.; Nicholas, D. D.; Schultz, T. P., Introduction to Wood Deterioration and Preservation. In Wood Deterioration and Preservation, American Chemical Society: 2003; Vol. 845, pp 2-7.

2. Mercer, T. G.; Frostick, L. E., Leaching characteristics of CCA-treated wood waste: A UK study. Science of the Total Environment 2012, 427-428, 165-174.

3. Vasishth, P.; Nicholas, D. D.; Henry, W. P.; Schultz, T. P., Effect of Polyvinyl Alcohol on Copper Leaching from Treated Wood. Forest Products Journal 2009, 59 (10), 28-30.

4. Moghaddam, A. H.; Mulligan, C. N., Leaching of heavy metals from chromated copper arsenate (CCA) treated wood after disposal. Waste Management 2008, 28 (3), 628-637.

5. Lupsea, M. O.; Mathies, H.; Schoknecht, U.; Tiruta-Barna, L.; Schiopu, N., Leaching from new generation treated wood: A chemical approach. WIT Transactions on Ecology and the Environment 2012, 162, 529-540.

6. Lupsea, M.; Tiruta-Barna, L.; Schiopu, N.; Schoknecht, U., Modelling inorganic and organic biocide leaching from CBA-amine (copper-boron-azole) treated wood based on characterisation leaching tests. Sci Total Environ 2013, 461-462.

7. Lupsea, M.; Mathies, H.; Schoknecht, U.; Tiruta-Barna, L.; Schiopu, N., Biocide leaching from CBA treated wood - A mechanistic interpretation. Science of the Total Environment 2013, 444, 522-530.

8. Tiruta-Barna, L.; Schiopu, N., Modelling inorganic biocide emission from treated wood. J Hazard Mater 2011, 192.

9. $\quad$ Schoknecht, U.; Kalbe, U.; van Zomeren, A.; Hjelmar, O., Laboratory leaching tests on treated wood according to different harmonised test procedures. Environmental Sciences Europe 2014, 26 (1).

10. Stook, K.; Tolaymat, T.; Ward, M.; Dubey, B.; Townsend, T.; Solo-Gabriele, H.; Bitton, G., Relative Leaching and Aquatic Toxicity of Pressure-Treated Wood Products Using Batch Leaching Tests. Environmental science \& technology 2005, 39 (1), 155-163.

11. Barnes, H. M., Wood: Preservative Treated. In Encyclopedia of Materials: Science and Technology Elsevier: Oxford, 2001; pp 9683-9688.

12. Schiopu, N.; Tiruta-Barna, L., Wood preservatives. In Toxicity of Building Materials, 2012; pp 138-165.

13. Morrell, J. J., 14 - Protection of Wood-Based Materials. In Handbook of Environmental Degradation of Materials, William Andrew Publishing: Oxford, 2012; pp 407-439.

14. Schultz, T. P.; Nicholas, D. D.; Preston, A. F., A brief review of the past, present and future of wood preservation. Pest Management Science 2007, 63 (8), 784-788.

15. Cookson, L. J., Preservative treatments suitable for hardwood window joinery. International Biodeterioration \& Biodegradation 2010, 64 (7), 652-658.

16. Schoknecht, U.; Wegner, R.; Horn, W.; Jann, O., Emission of biocides from treated materials test procedures for water and air. Environmental Science and Pollution Research 2003, 10 (3), 154-161.

17. Schoknecht, U.; Mathies, H.; Wegner, R.; Melcher, E.; Seidel, B.; Kussatz, C.; Maletzki, D., The Influence of Test Parameters on the Emission of Biocides from Preservative-Treated Wood in Leaching Tests. UFOPLAN 20367 441. Research report: BAM Berlin, 2004.

18. Schoknecht, U.; Mathies, H.; Morsing, N.; Lindegaard, B.; Sloot, H. A.; Zomeren, A.; Deroubaix, G.; Legay, S.; Tadeo, J. L.; Garcia-Valcárcel, A. I.; Gigliottti, G.; Zadra, C.; Hajšlová, J.; Tomaniová, M.; Wegner, R.; Bornkessel, C.; Fürhapper, C., Inter-laboratory Evaluation of Laboratory Test Methods to Estimate the Leaching from Treated Wood. European Grant Agreement no. 04/375757/C4, Report: BAM Berlin, 2005.

19. Woo, C. S.-M. Efficacy of tebuconazole and ddac in shell-treated wood. The University of British Columbia, Vancouver, 2010. 
20. Jaklová Dytrtová, J.; Fanfrlík, J.; Norková, R.; Jakl, M.; Hobza, P., Theoretical insight into the stabilization of triazole fungicides via their interactions with dications. International Journal of Mass Spectrometry 2014, 359 (1), 38-43.

21. Dytrtová, J. J.; Jakl, M.; Schröder, D.; Čadková, E.; Komárek, M., Complexation between the fungicide tebuconazole and copper(II) probed by electrospray ionization mass spectrometry. Rapid Communications in Mass Spectrometry 2011, 25 (8), 1037-1042.

22. Evans, P. D.; Schmalzl, K. J.; Forsyth, C. M.; Fallon, G. D.; Schmid, S.; Bendixen, B.; Heimdal, S., Formation and structure of metal complexes with the fungicides tebuconazole and propiconazole. Journal of Wood Chemistry and Technology 2007, 27 (3-4), 243-256.

23. Kennedy, M. J., Depletion of Copper-Based Preservatives from Pine Decking and Impacts on SoilDwelling Invertebrates. Florida Center for Environmental Solutions: Orlando, 2004; pp 124-145.

24. Kukowski, K.; Martinská, V.; Sedgeman, C. A.; Kuplic, P.; Kozliak, E. I.; Fisher, S.; Kubátová, A., Fate of triazoles in softwood upon environmental exposure. Chemosphere 2017, 184, 261-268.

25. Kukowski, K.; Martinská, V.; Krishnamoorthy, G.; Kubátová, A.; Kozliak, E., Diffusion of tebuconazole into softwood under ambient conditions and its distribution in freshly treated and aged wood. International Journal of Heat and Mass Transfer 2016, 102, 1257-1266.

26. De Meijer, M.; Militz, H., Moisture transport in coated wood. Part 2: Influence of coating type, film thickness, wood species, temperature and moisture gradient on kinetics of sorption and dimensional change. Holz als Roh - und Werkstoff 2001, 58 (6), 467-475.

27. Scown, D. K.; Cookson, L. J.; McCarthy, K. J.; Chew, N. Accelerated testing of window joinery made from eucalypts; 2004; $\mathrm{p} 45$.

28. Styszko, K.; Bollmann, U. E.; Bester, K., Leaching of biocides from polymer renders under wet/dry cycles - Rates and mechanisms. Chemosphere 2015, 138, 609-615.

29. EPA Woodlife 111 60061-103; Washington, D.C., 2014; p 2.

30. EPA PROVOST 433 SC; 264-861; Washington, D.C., 2008; 9.

31. EPA TOPSIN XTR; 73545-19; Washington, D.C., 2009; $p 10$. 